\title{
CONSIDERATIONS ANESTHESIQUES SUR L'HYPOPHYSECTOMIE TRANS-SPHENOIDALE: ETUDE RETROSPECTIVE
}

\author{
J. Côté et M. Rouitu.ARD
}

\section{INTRODUCTION}

L'APPROCHE TRANS-SPHÉNOIDALE de l'hypophyse connaît de nos jours une popularité croissante. Cette technique de par sa faible morbiditét-2 a supplanté la voie transfrontale. Ses indications ont été étendues, allant de la tumeur hypophysaire à l'hypophysectomie sélective pour enrayer l'évolution d'une rétinopathie diabétique ou d'une néoplasie hormono sensible et métastatique à l'os, telle celle du sein ${ }^{3-4}$ et de la prostate. Dans ce type de chirurgie, l'anesthésiste recontre des difficultés de deux ordres: celles découlant du patient et celles engendrées par la technique chirurgicale. ${ }^{5-6}$ Les patients conduits à la chirurgie sont d'âges variés et présentent des endocrinopathies de toutes sortes en période pré-opératoire et accusent des déficits hormonaux importants en période post-opératoire ${ }^{7}$. Le chirurgien travaille dans un champ restreint et utilise un appareillage encombrant ${ }^{5-6}$ contribuant à éloigner l'anesthésiste de son patient. Afin de mettre en évidence les principes qui ont guidé l'anesthésie, nous avons procédé à une étude rétrospective.

\section{Materiel Clinique}

Trente et un patients ont subi une hypophysectomie trans-sphénoidale dans notre milieu au cours des trois dernières années. Ce groups comprend 19 femmes et 12 hommes dont l'âge moyen est de 41.2 ans ( 9 à 63 ans).

Les différentes pathologies qui ont amené les malades à cette operation sont présentées au tableau I.

En regard du risque anesthésique selon la classification de l'A.S.A., 85 pour cent des patients ont été classifiés comme étant des risques I ou II, le reste étant des risques III.

Les interventions ont été pratiquées par la même équipe chirurgicale et la durée totale moyenne de l'anesthésie a été de 3 heures 54 minutes.

\section{Conduite Pre-Operatoire}

En plus des examens habituels, hémogramme, coagulagramme, examen d'urine, ionogramme, E.C.G. et radiographie pulmonaire, chaque patient a subi une investigation endocrinienne évaluant surtout la réserve surrénalienne et thyroidienne. L'étude radiologique comprend une radiographie du crâne, une pneumoencéphalographie et/ou une artériographie carotidienne. D’après les

Département d'Anesthésie-réanimation, Hôpital de l'Enfant-Jésus, Université Laval, Québec. 
TABLEAU I

RAISON DE L'INTERVENTION

\begin{tabular}{ll}
\hline & \\
\hline & $7 \%$ \\
Tumeur hypophysaire & $\mathbf{5 5}$ \\
Acromegalie & 15 \\
Microadenome & 10 \\
Hypophysectomie selective & 14 \\
pour douleur & \\
retinopathie diabetique & $\mathbf{6}$ \\
Syndrome de Cushing-Neilson & \\
\hline
\end{tabular}

TABLEAU II

REPARTITION DES PATIENTS SELON LE BILAN ENDOCRINIEN PRE-OPERATOIRE

\begin{tabular}{lr}
\hline \hline Bilan normal & \\
Bilan anormal & $22 \%$ \\
Alteration des gonadotropines & \\
Hormone de croissance $\uparrow$ & 25 \\
Hypocorticisme (primaire ou secondaire) & 12 \\
Hypothyroidie & 20 \\
Diabete & 9 \\
Secretion d'ADH $\uparrow$ & 4 \\
Pan hypopituitarisme & 4 \\
\hline
\end{tabular}

résultats de laboratoire, les patients ont été groupés selon le bilan endocrinien dont le détail apparaît au tableau II.

En vue de l'intervention, chaque patient recoit la veille et le matin de lintervention $50 \mathrm{mg}$ d'acétate de cortisone par voie intramusculaire; une perfusion d'hémisuccinate d'hydro-cortisone contenant $200 \mathrm{mg}$ dans $500 \mathrm{ml}$ de dextrose à 5 pour cent est également mis en route et ajusté à un débit de $75 \mathrm{ml} /$ hre le matin de l'intervention; une telle préparation en stéroide est d'ailleurs la mème dans tous les milieux..$^{7-12}$ De plus, on administre un antibiotique à large spectre depuis la veille de l'intervention jusqu'au septieme jour post-opératoire. Enfin, les patients ont prémédiqués de la façon suivante: diazepam $5-10 \mathrm{mg}$ ou flurazépam $30 \mathrm{mg}$ par voie orale la veille, puis le matin de l'intervention les patients ont reçu de la mépéridine suivis le matin de l'intervention de mépéridine à des doses variant de $50-100 \mathrm{mg}$ associée à atropine $0.4 \mathrm{mg}$ par voie intramusculaire une heure avant lintervention.

\section{Conduite Per-Operatoire}

La surveillance biologique per-opératoire comprend l'électrocardiogramme en continue, la mesure de la T.A. en continue ou à l'aide de l'artériosonde, la mise en place d'un Doppler à la région pré-cordiale, la diurèse horaire, la ventimétrie et la gazométrie.

Suivant le type d'induction, les malades sont classifiés en trois groupes: un premier représentant 60 pour cent des patients dont l'induction a été faite avec du thiopentone $3-5 \mathrm{mg} / \mathrm{kg}$ et précédée d'une dose protectrice de d-tubocurare. Une dose de succinylcholine $1 \mathrm{mg} / \mathrm{kg}$ fut administrée afin de faciliter l'intubation. Le deuxième groupe, soit 35 pour cent des malades, a reçu el même type d'induction 


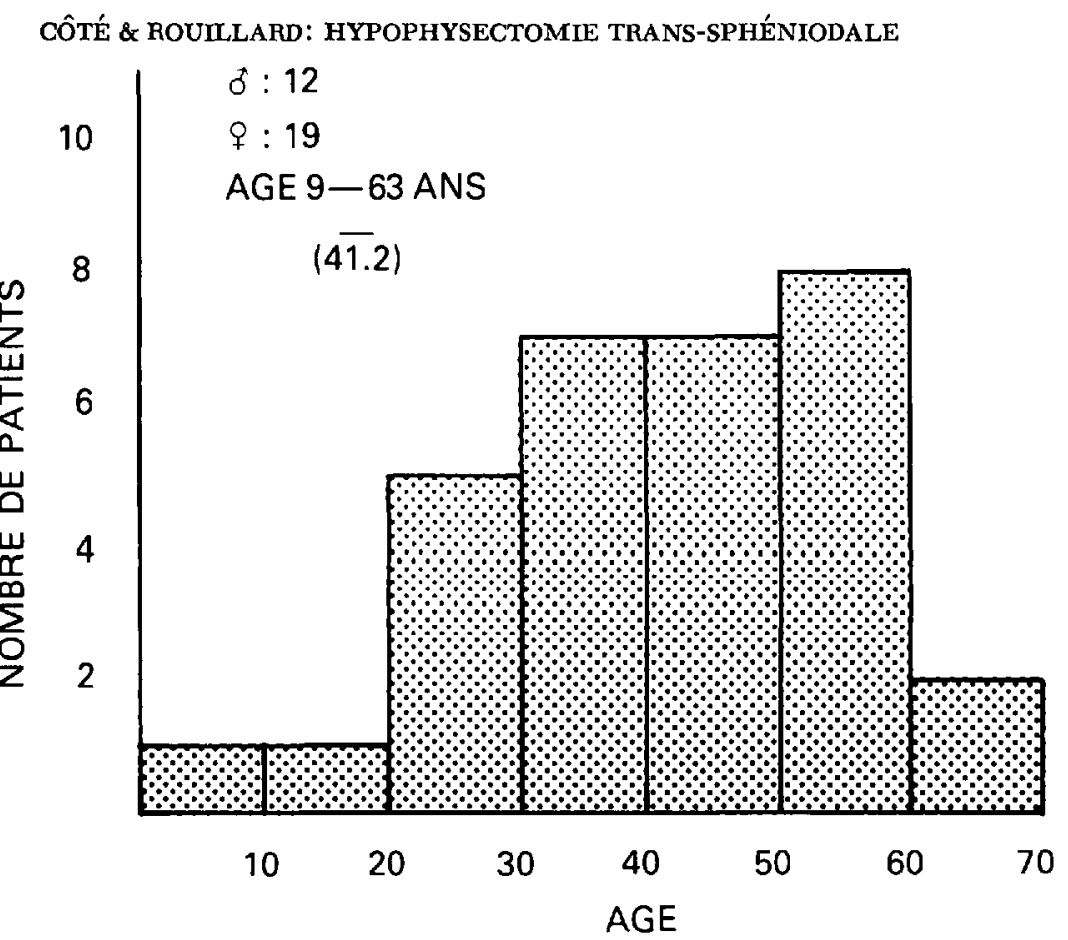

FIgure 1. Repartition des patients selon l'age.

mais précédé de 1-2 $\mathrm{ml}$ d'Innovar. ${ }^{\circledR}$ Enfin, deux malades ont reçu une induction par inhalation, soit un enfant de neuf ans et une femme de 38 ans, surrénalectomisée et hémiplégique à la suite d’un coma attribué à une insuffisance surrénalienne aigue.

L'intubation endotrachéale a été réalisée avec un tube jetable muni d'un ballonnet et suivie de la mise en place de compresses dans l'oropharynx. Ce type de tube s'est avéré adéquat et aucun incident n'a été rapporté. Lors de l'induction, il n'y a pas eu d'accident d'ordre cardiovasculaire ou d'ordre hypotensif. Nous n'avons relevé aucun problème d'intubation chez les acromégales, néanmoins, il faut souligner l'importance de l'évaluation de la cavité buccale lors de la visite pré-opératoire à cause d'eventuelles difficultés à l'intubation.

La respiration a été contrôlée chez 90 pour cent des patients, l'air courant étant maintenu à $11 \mathrm{ml} / \mathrm{kg}$, ce qui a été suffisant pour assurer en moyenne une $\mathrm{PaCO}_{2}$ de $35 \pm 5 \mathrm{~mm}$ de $\mathrm{Hg}$, soit une légère hypocapnie. Chez trois patients, la respiration a été assistée tandis qu'on a permis à un seul de respirer spontanément. Un relaxant musculaire a été employé pour faciliter la ventilation mécanique dans 70 pour cent des cas. Dans un des rares articles parus sur le sujet, Brunelle et Lepage ${ }^{10}$ ont préconisé l'utilisation de la respiration assistée prétextant que l'anesthésiste garde un contact plus étroit avec son patient, lui permettant ainsi de détecter rapidement toute anomalie respiratoire. Il nous a toutefois semblé que l'effet vasoconstricteur sur la circulation cérébrale, d'une légère hypocapnie représentait un avantage, de plus nous n'avons relevé aucun incident attribuable à cette technique.

Le maintien de l'anesthésie a été assuré avec du protoxyde d'azote, de l'oxygène et de fluothane chez 30 pour cent des patients, le méthoxyflurane a été employé 
chez 20 pour cent des patients, l'Innovar également utilisé chez 20 pour cent tandis que l'association de fluothane et d'Innovar a été employée chez 30 pour cent. Le méthoxyflurane fut utilisé exclusivement pour les premiers cas en raison de l'infiltration locale par le chirurgien d'une solution de lidocaine et d'épinéphrine. A toute fin pratique, le méthoxyflurane a été abandonné car il ne semble pas que ce soit l'anesthésique de choix à employer à cause des difficultés que pourrait poser le diagnostic différentiel entre la polyurie ${ }^{13-14}$ qu'il peut engendrer de celle associée au diabète insipide d'origine hypophysaire. L'halothane a été employé malgré l'usage d'épinéphrine, les précautions établies par Katz ${ }^{16}$ étant respectées. Cependant, l'incidence plus élevée des troubles du rythme cardiaque reliée à la chirurgie de cette région et l'emploi de l'halothane a eu pour conséquence d'en diminuer sa popularité comme agent anesthésique unique dans ce genre de chirurgie.

Pour ce qui est de la neuroleptanesthésie à l'Innovar, ${ }^{\circledR}$ certaines difficultés à maintenir les malades normotendus avec des doses permettant un réveil rapide ont amené les anesthésistes à supplémenter l'anesthésie avec de l'halothane, considérant l'effet hypotenseur de ce dernier comme étant un avantage dans ce type d'intervention. D'ailleurs, c'est dans cet optique que l'association de ces deux agents se justifie tout en permettant de conserver les avantages de l'anesthésie balancée.

La tension artérielle a été maintenue à des chiffres normaux chez 40 pour cent des patients au cours de l'anesthésie. Dans 30 pour cent des cas, elle a été abaissée de $10-20 \mathrm{~mm}$ de $\mathrm{Hg}$. Dans 30 pour cent des cas également, une baisse de $20-40 \mathrm{~mm}$ de $\mathrm{Hg}$ a été nécessaire. Enfin chez un patient seulement, il a fallu abaisser la tension arterielle (T.A.) systolique à $60 \mathrm{~mm}$ de $\mathrm{Hg}$ pour obtenir un champ chirurgical convenable, ce qui a été fait à l'aide d'un bloquant ganglionnaire.

Les pertes sanguines furent minimes à savoir moins de $100 \mathrm{ml}$ dans 60 pour cent des cas, entre 100 et $250 \mathrm{ml}$ dans 35 pour cent des cas, un patient a perdu $750 \mathrm{ml}$ et a dû être transfusé. Ce dernier présentait une tumeur hypophysaire d'allure maligne et une hydrocéphalie, le diagnostic histologique n'a pu être établi avec certitude. Nous avons essayé d'établir une corrélation entre le chiffre de la T.A. et les pertes sanguines. Parmi ceux qui ont perdu moins de $100 \mathrm{ml}, 55$ pour cent appartenaient aux groupes dont la T.A, a été maintenue sous la normale. Nous n'avons pas réussi à établir une corrélation entre les agents anesthésiques employés et l'importance de la perte sanguine. Il est intéressant de remarquer que les pertes sanguines ont été de moins de $100 \mathrm{ml}$ chez les patients laissés en respiration spontanée ou assistée. Enfin dans cette série, nous n'avons noté aucune corrélation entre le type de tumeur et les pertes sanguines.

Les complications per-opératoires ont été peu nombreuses. Trois patients ont présenté des arythmies cardiaques soit des extrasystoles ventriculaires survenant lors du temps nasal de l'intervention. Dans tous les cas, l'halothane était l'anesthésique utilisé pour l'entretien. Deux de ces patients ont nécessité comme traitement l'administration de lidocaine intraveineuse. Chez l'autre, une analgésie plus profonde a été suffisante pour faire disparaitre les troubles du rythme. Deux patients ont présenté une chute de la T.A. nécessitant l'emploi de vasopresseur. Pour le premier, il s'agissait d'un acromégale qui a présenté une chute de la T.A. à la fin de l'intervention après avoir reçu $2 \mathrm{ml}$ d'Innovar en raison d'une poussée 
hypertensive. Le deuxième patient opéré pour un syndrome douloureux relié à une néoplasie a présenté une chute de la T.A. au début de lintervention après une induction conventionnelle.

Mème si dans la littérature médicale ${ }^{8,10}$ on préconise l'utilisation de tube endotrachéal armé, nous n'avons relevé aucune complication chez nos patients à la suite de l'utilisation de tube endotrachéal conventionnel si ce n'est dans un cas où le patient a dû être réintubé durant l'intervention à cause d'une fuite importante provenant du ballonnet rendant impossible un contrôle ventilatoire adéquat.

\section{Conduite Post-Operatoire}

A la salle de réveil, les patients ont été suivis de façon uniforme. Six d'entre eux ont été laissés intubés parce que les réflexes de protection avaient été jugés inadéquats à leur arrivée. Vingt-cinq patients ont toutefois été extubés dans la salle d'opération, ces derniers pouvant répondre aux ordres simples dès ce moment. Tous les patients ont été installés en position demi-assise afin d'éviter la congestion veineuse au niveau du champ opératoire. Ils ont, de plus, reçu une dose standard de $50 \mathrm{mg}$ d'acétate de cortisone par voie intraveineuse. Le monitoring des paramètres cardio-respiratoires, de la température et de la diurèse horaire a été effectué.

\section{Complications Post-Operatorres}

Au cours de la période post-opératoire immédiate, un patient a été ventilé mécaniquement à la Salle de réveil en raison d'une dépression respiratoire, l'anesthésie ayant été maintenue avec du méthoxyflurane. Cependant, ce patient a pu être extubé une heure plus tard. L'incidence de nausée et de vomissement a été faible; de plus l'emploi d'analgésique n'a pas été nécessaire. Aucune hémorragie n'a été rapportée. Un patient reconnu hypertendu a présenté une poussée hypertensive nécessitant un traitement.

A plus long terme, 70 pour cent des patients ont présenté un diabète insipide ${ }^{5,11,15-23}$ apparaissant dans le premier vingt-quatre heures et nécessitant l'injection intra-musculaire de tannate de pitressine pour une durée allant de un à six jours. Un patient a présenté un tableau clinique de panhypopituitarisme amélioré par l'emploi de stéroide et de thyroxine. Un autre a présenté, au cours des premiers jours qui ont suivi l'intervention, un épisode convulsif attribué à une intoxication à l'eau. Aucune autre complication n'a été rapportée et aucun décès n'a été constaté.

\section{Conclusion}

L'hypophysectomie par voie trans-sphénoidale constitue un défi que l'anesthésiste peut relever avec succès en observant certains principes qui ressortent de cette étude. Une investigation élaborée assure un diagnostic certain et établit le bilan endocrinien de chaque patient. L'administration d'agents stéroidiens en pré et post-opératoire permet aux patients d'affronter en sécurité le stress relié à la chirurgie et à l'anesthésie. Au point de vue anesthésie, il ressort de cette étude qu'une induction conventionnelle s'est avérée efficace. Il est de prime importance 
d'assurer un airway à toute épreuve et pour ce faire, le tube endotrachéal doit être d'une part indélogeable, d'autre part, il nous est apparu important de renforcer son étanchéité par la mise en place de compresses pharyngées étant donné l'emploi de solution d'irrigation et des pertes sanguines. La respiration contrôlée en raison de l'hypocapnie légère qu'elle produit et de ses effets sur la circulation cérébrale semble justifiée. Une anesthésie balancée utilisant la combinaison de fluothane et d'Innovar ${ }^{\circledR}$ nous paraît indiquée dans une telle intervention, permettant le maintien d'une T.A. stable ou diminuée si besoin. Une surveillance basée sur les dangers potentiels de cette intervention soit une hémorragie importante, le besoin d'hypotension et la possibilité lointaine d'embolie aérienne, doit comprendre un cathéter à pression veineuse centrale, un Doppler à la région pré-cordiale, une mesure adéquate de la T.A. par une canulation, la gazométrie et la diurèse horaire. Des suites opératoires sans complication sont à prévoir si un support endocrinien adéquat basé sur le remplacement stéroidien et le traitement du diabète insipide est donné à chaque malade.

\section{RÉSUMÉ}

L'hypophysectomie trans-sphénoidale étant une procédure de plus en plus utilisée, les auteurs ont voulu élaborer sur la conduite anesthésique à partir d'une étude rétrospective chez 31 patients.

Il ressort de cette étude les points suivants: un diagnostic précis appuyé par une investigation endocrinienne complète doit être établi dans tous les cas. Une préparation adéquate au moyen de stéroides ainsi que l'administration per-opératoire de ces substances s'imposent. En ce qui regarde la conduite anesthésique, les auteurs soulignent l'importance d'un monitoring adéquat, d'un airway sécuritaire et à toute épreuve. Ils préconisent l'association Innovar et halothane pour le maintien de l'anesthésie et ne voient pas l'intérêt de procéder à de l'hypotension délibérée de façon routinière. Ils pensent qu'une légère hypocapnie est préférable. Enfin, ils concluent que la période per-opératoire est dépourvue d'incidents majeurs si ces principes sont suivis; cependant, le diabète insipide est la règle au cours de la période post-opératoire et par conséquent, une surveillance étroite doit être faite.

\section{Summary}

This paper deals with the retrospective analysis of anaesthetic management of 31 cases of trans-sphenoidal hypophysectomy.

Good management relies on the following points:

(1) a precise diagnosis supported by thorough hormonal investigation,

(2) adequate pre- and per-op. administration of steroids,

(3) adequate monitoring,

(4) a fail-safe airway.

The authors recommend the combined use of Innovar and halothane under light

hypocapnea for the maintenance of anaesthesia. The routine use of deliberate hypotension is not indicated.

Diabetes insipidus usually results from this operation and consequently must be treated. 


\section{BIBLIOGRAPHIE}

1. Guiot, G. \& Derome, P. Les indications de la voie d'abord trans-sphénoidale en neurochirurgie. Expérience de 521 interventions. Ann. Med. Int., 123, 8-9: 703-12 (1972).

2. Reyes-Oliveros, M.F. Considérations sur la chirurgie trans-sphénoidale de l'hypophyse. Neurochirurgie, 17, 3: 209-15 (1971).

3. Hardy, J., Leclerce, T.A., \& Somma, M. Le traitement du cancer du sein métastatique par l'hypophysectomie transsphénoidale. Expérience de 160 cas. L’Un. Méd. du Can., 104: 1557-62 (1975).

4. Robin, P.E., Powell, J., \& Waterhouse, J.A.H. Transsphenoidal hypophysectomy in disseminated carcinoma of the breast. Brit. J. Surg., 62, 2: 85-91 (1975).

5. Hardy, J. La chirurgie de l'hypophyse par voie transsphénoidale, étude comparative de deux modalités techniques. L'Un. Méd. du Can., 96: 702-12 (1967).

6. HaRDY, J. Transsphenoidal hypophysectomy. J. Neurosurg. 34: pp. 582-94 (1971).

7. Ezrin, C., Briant, T.D.R., Figestone, G., \& Rosen, F. The endocrine aspects of transsphenoidal hypophysectomy. Can. Med. Ass. J. 97: 72-74 (1967).

8. MCComish, P.R. and BodLEY, P.O. Anaesthesia for neurological surgery. Year Book Medica Publ., Chicago, pp. 200-17 (1971).

9. Wylie, W.D. \& Churchill-Davidson, H.C. A practice of anaesthesia. Year Book Med. Publishers, Chicago, pp. 1363 ( 1971 ).

10. Brunelle, J.P. \& Lepage, C. L'Anesthésie dans la chirurgie hypophysaire transsphénoidale, L'Union Méd. Cans. 93: 84-85 (1964).

11. Kapur, T.R. \& Dalton, G.A. Transsphénoidal hypophysectomy for metastatic carcinoma of the breast. Brit. J. Surg. 56, 5: 332-37 (1969).

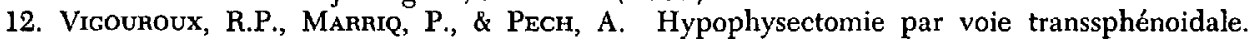
Marseille Med., 109, 5: 347-58 (1972).

13. Mazze, R.I., Shue, G.L., \& Jackson, S.H. Renal dysfunction associated with methoxyflurane anesthesia. J.A.M.A. 216, 2: 278-88 (1971).

14. Cousins, M.J., Mazze, H.I., \& Kosek, J.C. The ethiology of methoxyflurane nephrotoxicity. J. Pharmacol, Exp. Ther. 190: 530-41 (1974).

15. Bouche, J., Guiot, G., \& Freche, Ch. Evaluation de l'hypophysectomie par voie transsphénoidale basse après 120 interventions. Ann. O.R.L. (Fr) 83, 6: 466-71 (1966).

16. KATZ, R.L. \& EPSTEIN, R.A. The interaction of anesthetic agents and adrenergic drugs to produce cardiac arythmias. Anesthesiology 4: 763-84 (1968).

17. Goutelle, A., Hagvenauer, J.P., \& Chahachon, R. La chirurgie transsphénoidale des tumeurs de la loge hypophysaire. J. F. ORL. XV, 8: 1007-15 (1966).

18. Bouche, J. L'abord transsphénoidal de l'hypophyse, technique et médicaments. La Rcvue du Praticien, Tome XII, 21 : 2329-32 (1962).

19. Handy, J., PAnisset, A., \& LANThier, A. Microsurgical selective anterior pituitary ablation for diabetic retinopathy. Can. Med. Ass. J. 100, 17; 785-92 (1969).

20. HARDY, J. L'exérèse des adénomes hypophysaires par voie transsphénoidale. L'Un. Méd. du Can. 91 : 933-45 (1962).

21. Speakman, J.S., Murtimer, C.B., Briant, T.D.R., Ezrin, C., Lougheed, N.N., \& Clafke, W.T. Pituitary ablation for diabetic retinopathy. Can. Med. Ass. J. 94, 13: 627-35 (1966).

22. Hudson, W.R. \& KENAN, P.D. Transsphénoidal management of pituitary adenomas and other selected lesions of the sella turcica. Laryngoscope, 84: 1159-69 (1974).

23. Cirig, I.S. \& Tahkington, J. Transsphénoidal microsurgery. Surg. Neurol. 2: 207-12 (1974). 